\title{
Off-axis injection for storage rings with full coupling
}

\author{
Tong Zhang ${ }^{1,2}$ and Xiaobiao Huang, ${ }^{2, *}$ \\ ${ }^{1}$ University of Science and Technology of China, 96 Jinzhai Rd, Hefei, Anhui 230000, China \\ ${ }^{2}$ SLAC National Accelerator Laboratory, 2575 Sand Hill Road, Menlo Park, California 94025, USA
}

(Received 8 June 2018; published 28 August 2018)

\begin{abstract}
The next generation of storage ring light sources will likely operate with high linear coupling, which could potentially prevent the use of off-axis injection as large horizontal oscillation of the injected beam is coupled to the vertical plane. We did experiments on the SPEAR3 storage ring to study how linear coupling impact the dynamic aperture and the off-axis injection efficiency. The results show that the dynamic aperture is significantly reduced and injection efficiency can drop to zero when operated on the coupling resonance. However, with large nonlinear detuning, the dynamic aperture and high injection efficiency can survive with the stored beam at full coupling because the injected beam is shifted away from the coupling resonance. Particle tracking simulation is also conducted to demonstrate the injected beam behavior in a nonlinear lattice with high coupling.
\end{abstract}

DOI: 10.1103/PhysRevAccelBeams.21.084002

\section{INTRODUCTION}

In existing 3rd generation light sources, the vertical emittance is typically much smaller than the horizontal emittance, with an emittance ratio below $1 \%$. However, for future diffraction limited storage rings (DLSRs), it is not advisable to maintain the same level of vertical-tohorizontal emittance ratio. This is because the horizontal emittance will already be diffraction-limited and hence there is no need to make vertical emittance any smaller, and small vertical emittance will cause significant intrabeam scattering (IBS) and Touschek beam loss. A round beam with equal horizontal and vertical emittances is usually assumed in DLSR designs, and most designs plan to generate round beams by operating on the linear coupling resonance, with fully coupled horizontal and vertical motion. This would be acceptable if on-axis, swap-out injection $[1,2]$ will be used. However, for the DLSRs that plan to use the traditional off-axis injection scheme, such as PEP-X [3] and PETRA-IV [4], large coupling between the two transverse planes could cause injection difficulties. With off-axis injection, the injected beam will have large vertical oscillation amplitude through coupling and the initial horizontal offset and may get lost to the small vertical apertures such as the small-gap insertion devices. While other schemes to generate round beams in storage rings have been proposed [5-8], the approach of operating on the

\footnotetext{
*xiahuang@slac.stanford.edu
}

Published by the American Physical Society under the terms of the Creative Commons Attribution 4.0 International license. Further distribution of this work must maintain attribution to the author(s) and the published article's title, journal citation, and DOI. coupling resonance is still a major contestant in DLSR ring design studies.

Off-axis injection with high coupling has been experimentally studied in Refs. [9-11]. Reference [9] showed that at high coupling the injection efficiency dropped to zero. In Ref. [10] it was shown that if the coupling resonance strength is corrected to a low level the injection efficiency does not suffer a loss as the coupling resonance is crossed. To understand the dynamics of beam motion near the coupling resonance and to investigate its potential impact to off-axis injection, we conducted similar experiments on a storage ring. Our experiments revealed the causes of high or no losses during off-axis injection with high coupling, which are rooted in the nature of nonlinear oscillation of a coupled system.

In the experiments we changed the strength of the linear difference coupling resonance. At each level we moved the horizontal and vertical tunes toward each other to cross the coupling resonance, which changed the coupling ratio. Dynamic aperture and injection efficiency were measured as the betatron tunes were crossed. In the measurements we also observed safe crossing of the linear coupling resonance $\left(\nu_{x}-\nu_{y}=\right.$ integer for the stored beam) when the coupling strength was corrected to a low level. However, the dynamic aperture and injection efficiency suffered significant loss at a point after the crossing, which then recovered as the betatron tunes moved further apart.

An explanation based on the nonlinear detuning of the injected beam is given for the experimental observation. Particle tracking simulation is done to demonstrate the beam motion of injected beam under the experimental conditions. The simulation clearly shows that when the stored beam is on the linear difference coupling resonance, the injected beam is not on the resonance due to nonlinear 
TABLE I. Main parameters of SPEAR3.

\begin{tabular}{lc}
\hline \hline Parameters (unit) & Values \\
\hline Beam Energy (GeV) & 3 \\
Current $(\mathrm{mA})$ & 500 \\
Circumference $(\mathrm{m})$ & 234.1 \\
rf Frequency (MHz) & 476.3 \\
Horizontal Emittance (nm rad) & 10 \\
Vertical Emittance $(\mathrm{pm} \mathrm{rad})$ & 10 \\
Betatron Tunes $\left(\nu_{x}, \nu_{y}\right)$ & $(14.106,6.177)$ \\
\hline \hline
\end{tabular}

tune shifts with oscillation amplitude, and hence the horizontal oscillation is not strongly coupled to the vertical plane. On the other hand, at a certain working point when the stored beam is not on the coupling resonance, the injected beam could be right on the coupling resonance, which causes the horizontal oscillation to couple to the vertical plane to cause beam losses.

The finding of this study could serve as a guide for the design of DLSR lattices with off-axis injection. It may be possible to tailor the tune footprint of the injected beam to avoid the linear difference resonance, while keeping the stored beam working point on resonance to generate round beam.

The experimental and simulation studies are based on the SPEAR3 storage ring. The main parameters of SPEAR3 are listed in Table I.

In the following we will first briefly discuss the theory of beam motion with linear coupling in Sec. II. A detailed description of the experimental setup and results are shown in Sec. III. Simulation results are presented in Sec. IV. The conclusion is given in Sec. V.

\section{LINEAR COUPLING MOTION}

The Hamiltonian for the coupled beam motion between the horizontal and vertical planes due to skew quadrupole fields is given by

$H=\frac{1}{2} x^{\prime 2}+\frac{1}{2} y^{\prime 2}+\frac{1}{2} K_{x}(s) x^{2}+\frac{1}{2} K_{y}(s) y^{2}-a_{1}(s) x y$,

where $a_{1}=\frac{1}{B \rho} \frac{d B_{x}}{d x}$ is the skew quadrupole gradient, and the last term, $H_{1}=-a_{1}(s) x y$, can be considered a perturbation to the otherwise uncoupled linear motion. By taking a few canonical transformations toward the action-angle coordinates, $\left(J_{x}, \phi_{x}\right)$, expanding the perturbation term in Fourier series, and keeping only the term with slow varying phase factor near the linear difference resonance $\nu_{x}-\nu_{y}=l$, we arrive at a new Hamiltonian [12]

$\bar{H}=\nu_{x} J_{x}+\nu_{y} J_{y}+G \sqrt{J_{x} J_{y}} \cos \left(\phi_{x}-\phi_{y}+\chi-l \theta\right)$,

where $\theta=s / R, R$ is the ring radius and

$$
G e^{i \chi}=-\frac{1}{2 \pi} \oint a_{1}(s) \sqrt{\beta_{x} \beta_{y}} e^{i \Psi_{-}} e^{-i l \theta} d s
$$

with $\Psi_{-}=\psi_{x}-\psi_{y}-\left(\nu_{x}-\nu_{y}\right) \theta, \psi_{x, y}=\int_{0}^{s} \frac{1}{\beta_{x, y}\left(s^{\prime}\right)} d s^{\prime}$, and $\beta_{x, y}$ are beta functions.

Taking another canonical transformation to the resonant precessing frame with the generating function [12]

$$
F_{2}\left(\phi_{x}, \phi_{y}, J_{1}, J_{2}\right)=\left(\phi_{x}-\phi_{y}+\chi-l \theta\right) J_{1}+\phi_{y} J_{2}
$$

and new coordinates

$$
\begin{gathered}
\phi_{1}=\phi_{x}-\phi_{y}+\chi-l \theta, \quad J_{1}=J_{x}, \\
\phi_{2}=\phi_{y}, \quad J_{2}=J_{x}+J_{y},
\end{gathered}
$$

the Hamiltonian becomes

$$
\begin{aligned}
& \tilde{H}=H_{1}\left(J_{1}, \phi_{1}, J_{2}\right)+H_{2}\left(J_{2}\right), \quad \text { with } \\
& H_{1}=\Delta J_{1}+G \sqrt{J_{1}\left(J_{2}-J_{1}\right)} \cos \phi_{1},
\end{aligned}
$$

and $H_{2}=\nu_{y} J_{2}$, with $\Delta=\nu_{x}-\nu_{y}-l$.

If a particle is launched initially from a horizontal plane with action variable $J_{1, \max }=J_{2}$, from Eq. (8) we obtain

$$
G^{2} J_{1} \cos ^{2} \phi_{1}=\Delta^{2}\left(J_{2}-J_{1}\right)
$$

which leads to the maximum action value of the vertical action variable

$$
J_{y, \max }=J_{2} \frac{G^{2}}{\Delta^{2}+G^{2}} .
$$

The ratio of maximum action variables for the two planes is found to be

$$
\frac{J_{y, \max }}{J_{x, \max }}=\frac{G^{2}}{\Delta^{2}+G^{2}}
$$

The ratio between the actions in the two planes is the same as the emittance ratio, $\epsilon_{y} / \epsilon_{x}$, since betatron coupling is typically the dominant source of vertical emittance. The emittance ratio is often also referred to as the coupling ratio. The coupling ratio increases with the coupling strength $G$ and becomes $100 \%$ on the difference coupling resonance, when $\Delta=0$.

\section{THE EXPERIMENTS}

We did experiments to study the impact of high linear coupling to the injection efficiency on SPEAR3. The nominal working point is at [14.106, 6.177]. The injected beam comes into SPEAR3 at the end of a Lambertson septum magnet with a horizontal separation from the stored 


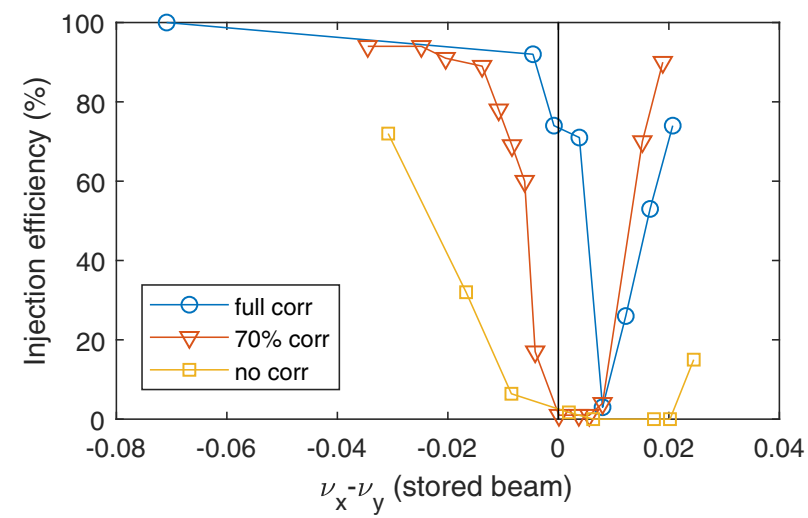

FIG. 1. Injection efficiency vs tune separation at three levels of linear difference resonance strengths.

beam. The distance between the centers of the injected beam and the stored beam during injection is $11 \mathrm{~mm}$.

During normal operation the coupling ratio is corrected to about $0.1 \%$, using 13 skew quadrupole magnets. In the experiments we changed the strength of the difference coupling resonance by the same skew quadrupole magnets. We first used LOCO [13], the orbit response matrix based method, to correct linear coupling to a low level. The coupling resonance strength was then changed by scaling the skew quadrupole setpoints to $70 \%$ of the full correction values, or turning the skew quadrupoles off. A lattice model was obtained with LOCO fitting for each case. Additional skew quadrupole variables in the model were used in the fitting to more accurately represent the coupling information in the orbit response matrices. At the nominal working point, the coupling ratio was $0.07 \%$ with full correction, $0.13 \%$ with skew quadrupoles at $70 \%$ strengths, and $0.95 \%$ with skew quadrupoles off, respectively.

At each of the three levels of coupling resonance strengths, the horizontal and vertical tunes were moved toward each other in equal steps. In this process the tune separation, $\Delta=\nu_{x}-\nu_{y}-8$, moved from negative territory toward the resonance condition, $\Delta=0$, and over to the positive side. As the working point moves toward the difference resonance line, the coupling ratio increases. The injection efficiency and the dynamic aperture were measured at each step.

Figure 1 shows the injection efficiency as a function of $\Delta$ for the three cases. With skew quadrupoles off ("no corr"), there is a total loss of injection efficiency over a wide range of tune separation about the resonance condition. With skew quadrupoles powered at $70 \%$ of the correction values ("70\% corr"), as the tune separation approaches $\Delta=0$ from below (i.e., initially $\Delta<0$ ), the injection efficiency decreases, reaching zero near the resonance condition. The injection efficiency stays at zero within a small region, then almost completely recovers when the tune separation moves further to the positive territory. The width of the region with total injection efficiency loss (between $\Delta=$ -0.006 and 0.014 ) is much smaller than the "no corr" case.

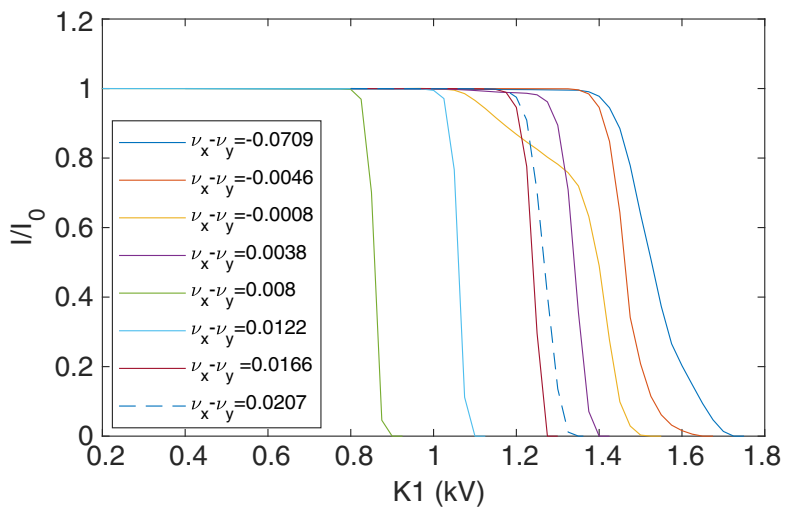

FIG. 2. Dynamic aperture measurements with full coupling correction.

The center of this region is shifted toward the positive direction.

When the skew quadrupoles are at the full correction strength ("full corr"), the region with loss of injection efficiency is even smaller. For this case, when the betatron tunes are moved on the resonance condition, the injection efficiency is still at about $80 \%$. However, as the tune separation moves away from the resonance condition, at $\Delta=0.008$, the injection efficiency drops to nearly zero. The injection efficiency also recovers as the tune separation moves further up.

We measured the dynamic aperture by kicking a short train of stored beam bunches until the beam was lost with one injection kicker. Figure 2 shows the dynamic aperture measurements for the case with full skew quadrupole correction, where the fraction of surviving beam is plotted against the kicker voltage. We may define dynamic aperture arbitrarily as the kicker voltage when $80 \%$ of the initial beam current is lost. Figure 3 shows the measured dynamic aperture at various tune separations for the three cases. Roughly speaking, a voltage of $1 \mathrm{kV}$ corresponds to a dynamic aperture of $11 \mathrm{~mm}$ for this lattice. The dynamic aperture measurements generally agree with the injection

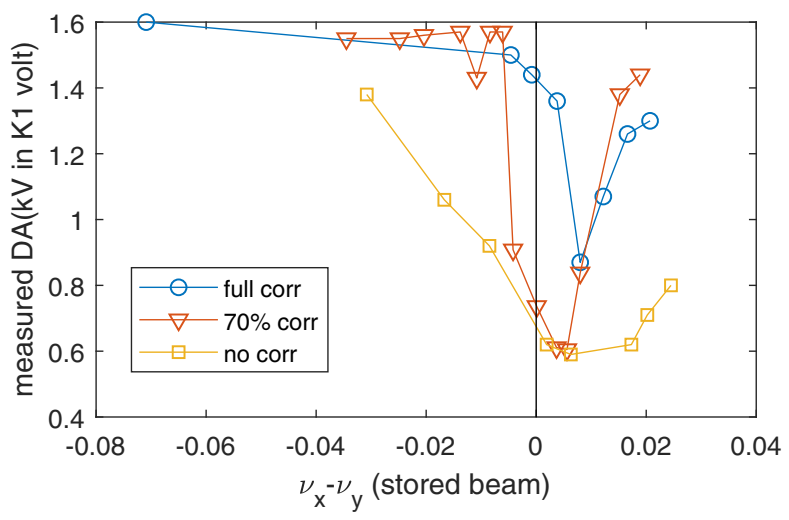

FIG. 3. Dynamic aperture vs tune separation for the three coupling levels. 


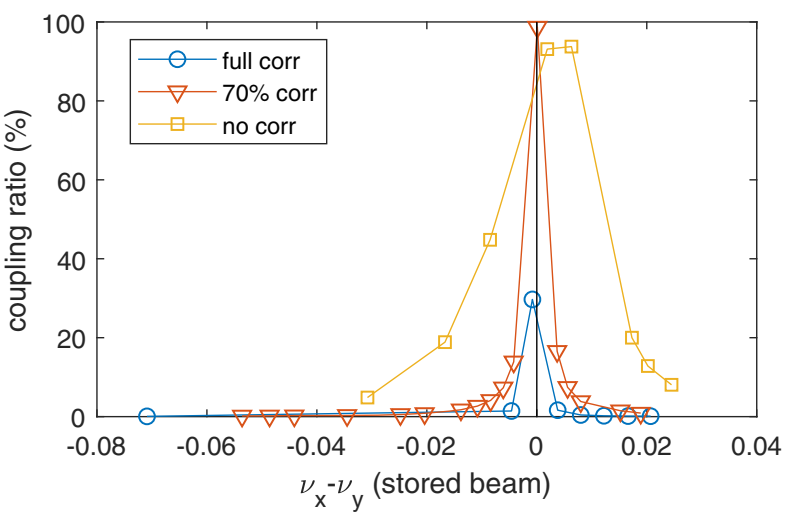

FIG. 4. Coupling ratio (stored beam) for the three cases.

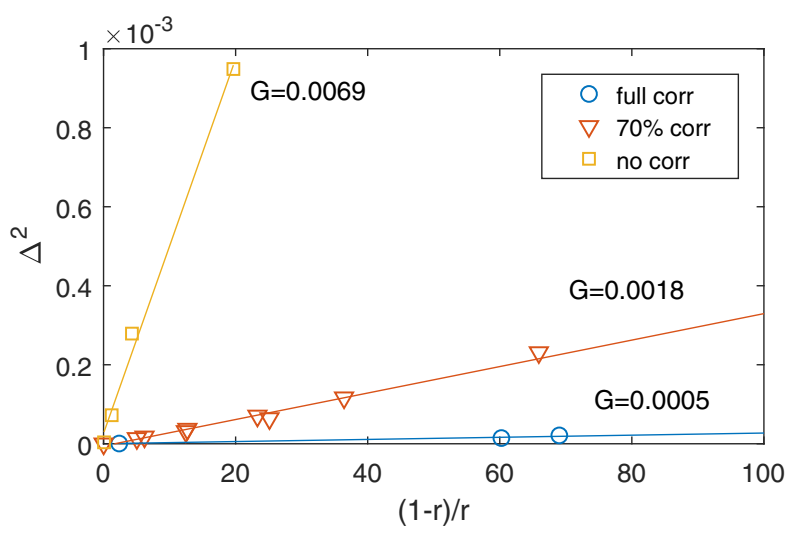

FIG. 5. Fitting coupling ratio $\left(r \equiv \frac{\epsilon_{y}}{\epsilon_{x}}\right)$ and tune separation data to obtain the coupling resonance strength coefficient $G$.

efficiency measurements. For the case with minimum coupling strength ("full corr"), the dynamic aperture is on an acceptable level when the working point is nearly on resonance $(\Delta=-0.0008$ and 0.0038$)$, while suffering a significant reduction at $\Delta=0.008$.

Using the lattice models obtained with LOCO, we can calculate the coupling ratios, which are shown in Fig. 4. Using Eq. (11), we can fit the coupling ratio vs. $\Delta$ data to obtain the coupling resonance strength coefficient $G$. The data fitting is shown in Fig. 5. The coupling strength coefficient is $G=0.52 \times 10^{-3}, 1.83 \times 10^{-3}$, and $6.89 \times 10^{-3}$ for the three cases, respectively, with the lowest value for the full correction case.

\section{SIMULATION}

The observation we made in the experiments appears to be puzzling at first sight: why is the injection efficiency significantly reduced when the working point is not on the difference resonance, and not much reduction occurs when the beam is right on resonance? A close examination indicates that this could be explained by the differences between the betatron tunes of the stored beam and the injected beam due to nonlinear detuning by the horizontal oscillation of the latter.

Nonlinear detuning coefficients for SPEAR3 have been measured previously [14]. The tune shift with amplitude coefficients due to horizontal oscillation are $\frac{d v_{x}}{d\left(2 J_{x}\right)}=$ $1590 \mathrm{~m}^{-1}$ and $\frac{d v_{y}}{d\left(2 J_{x}\right)}=2200 \mathrm{~m}^{-1}$, respectively, where $2 J_{x}=x^{2} / \beta_{x}$. The initial offset of the injected beam is $x=$ $11 \mathrm{~mm}$ and the horizontal beta function at the septum is $\beta_{x}=9.0 \mathrm{~m}$, from which the tune shifts of the injected beam are calculated to be $[0.0214,0.0296]$. Therefore, when the stored beam is on the linear difference coupling resonance, the injected beam has a tune separation of $\Delta=-0.0082$, which would shift the injected beam off the resonance when the coupling resonance strength is corrected to a low level.

We conducted particle tracking simulation to investigate the injected beam behavior in a nonlinear lattice at or near the linear difference coupling resonance. As discussed in Ref. [14], the tune shift with amplitude coefficients for the nominal SPEAR3 lattice model are slightly different from the values measured on the actual machine. To reproduce the lattice condition in the model, we introduced an artificial element to change the tune shifts with amplitude. In this element, the particle phase space coordinates $\left(x, p_{x}\right)$ are first transformed to the normalized coordinates, $\left(\tilde{x}, \tilde{p}_{x}\right)$, with

$$
\left(\begin{array}{c}
\tilde{x} \\
\tilde{p}_{x}
\end{array}\right)=\left(\begin{array}{cc}
\frac{1}{\sqrt{\beta}} & 0 \\
\frac{\alpha}{\sqrt{\beta}} & \sqrt{\beta}
\end{array}\right)\left(\begin{array}{c}
x \\
p_{x}
\end{array}\right) .
$$

This is followed by a rotation transformation to the normalized coordinates that accounts for the tune differences due to the oscillation amplitude. Finally the updated normalized coordinates are transformed back to the $\left(x, p_{x}\right)$ coordinates using the inverse transformation of Eq. (12). The same procedure is applied to the vertical coordinates. Particle tracking is done with the accelerator modeling code Accelerator Toolbox [15].

It is worth pointing out that the discrepancy in the amplitude dependent tune shifts between the lattice model and measurements can be reduced by refining the lattice model. At SPEAR3, we built a lattice model that integrates the particle motion through the magnetic fields of dipole and quadrupole magnets to include fringe field effects and the effect of a combined-function rectangular dipole [16]. This model has good agreement with the measurements. It is not used in this study because the integration is not symplectic and thus not suitable for long term tracking. However, it is possible to build symplectic models that include these effects. For future DLSRs, it is essential to use refined lattice models in the design optimization in order to have reliable prediction of the nonlinear beam dynamics performance.

Figure 6 shows the changing betatron tunes of the injected beam after injection in the tune diagram for two 


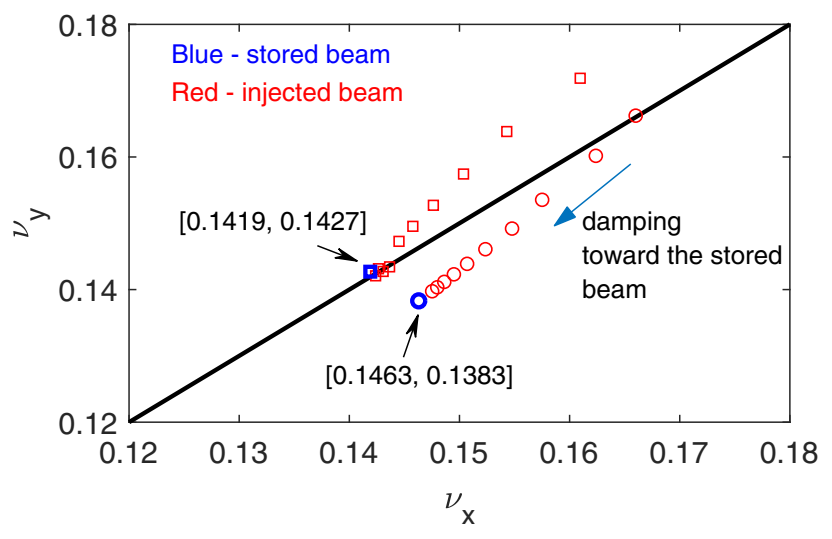

FIG. 6. Changing betatron tunes for the injected beam for the full coupling correction case when the stored beam tunes are $[0.1419,0.1427]$ (squares) or [0.1463, 0.1383] (circles).
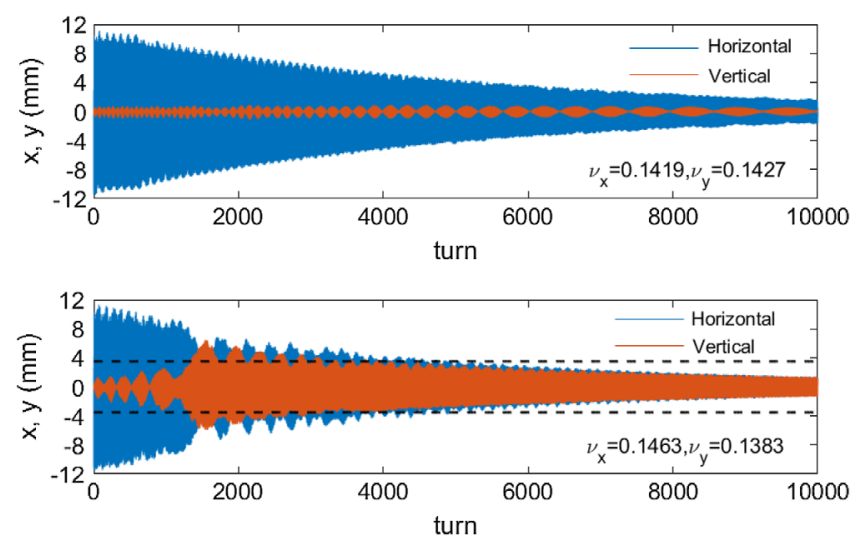

FIG. 7. Horizontal and vertical oscillations for the injected beam for the same two cases as in Fig. 6. Top: $\left[\nu_{x}, \nu_{y}\right]=$ [0.1419, 0.1427]; bottom: $\left[\nu_{x}, \nu_{y}\right]=[0.1463,0.1383]$. Dashed lines in the bottom plot show the physical aperture at $\pm 3.5 \mathrm{~mm}$.

lattice working points for the case with full coupling correction $(G=0.00052)$. The two working points are $\left[\nu_{x}, \nu_{y}\right]=[0.1419,0.1427]$ and $[0.1463,0.1383]$, respectively. The first working point corresponds to the experimental data point when the stored beam is almost on the difference coupling resonance. The second working point corresponds to the data point when the injection efficiency dropped to nearly zero (see Fig. 1). The betatron tunes of the injected beam change as its oscillation amplitude decreases due to radiation damping. The time interval between the red data points is 1000 turns.

Figure 7 shows the injected beam oscillations in both transverse planes with time for the same two cases. The observation point is the septum magnet, where the beta functions are $\beta_{x}=9.0 \mathrm{~m}$ and $\beta_{y}=5.3 \mathrm{~m}$. In the first case (top plot), not much vertical oscillation is excited on the injected beam because the nonlinear betatron tunes are sufficiently separated at the time of injection, when there is a large horizontal oscillation amplitude. In the second case

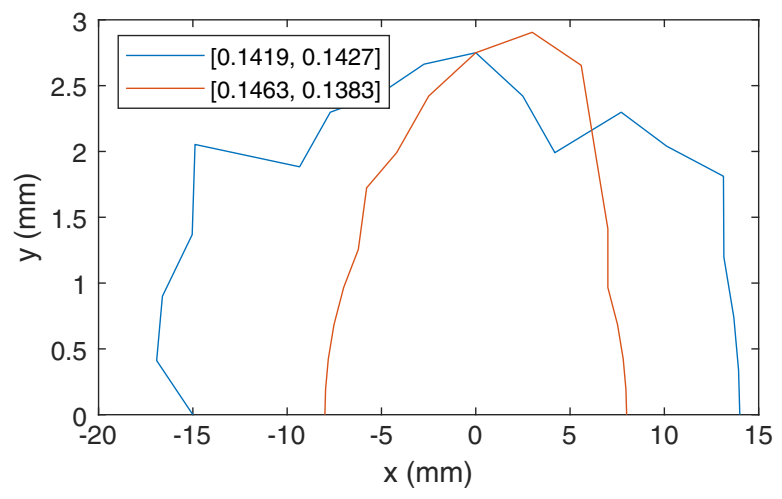

FIG. 8. Dynamic aperture from particle tracking simulation for the same two working points as Fig. 6 for the minimum coupling case.

(bottom plot), however, pronounced vertical oscillation is excited through the horizontal-to-vertical coupling, as the tunes of the injected beam satisfy the linear difference resonance condition. The vertical oscillation amplitude exceeds the physical aperture (dark dashed lines), which means the particle would be lost during injection.

We also used particle tracking to determine the dynamic aperture of the lattice for the two cases discussed in the above. The vertical physical aperture is included in the model. The results (see Fig. 8) are consistent with the experimental observations and the above discussion. The dynamic aperture for the case with $\left[\nu_{x}, \nu_{y}\right]=[0.1419,0.1427]$ is close to that of the nominal operation lattice, even though the linear betatron tunes are nearly on the difference resonance. The dynamic aperture for the other case, with $\left[\nu_{x}, \nu_{y}\right]=[0.1463,0.1383]$, however, suffers a substantial loss on the horizontal plane.

The simulation results provide a clear explanation of the experimental observations. In the SPEAR3 case, the $\nu_{x}$ and $\nu_{y}$ nonlinear detuning coefficients with respect to horizontal oscillation are different, which shift the nonlinear betatron tunes away from the difference coupling resonance condition for the injected beam. This is why we can have good injection with the linear tunes on the resonance. As the linear tunes are changed to the point that puts the nonlinear betatron tunes on the difference resonance, the dynamic aperture is substantially reduced and the injected beam is lost due to the vertical oscillation coupled from the horizontal plane.

\section{CONCLUSION}

Our experiments on the SPEAR3 storage ring showed that the injected beam that comes in off-axis with an initial horizontal separation can be coupled to the vertical plane and get lost to the small vertical aperture, even when the linear difference coupling resonance is corrected to a low level. This happens when the actual betatron tunes of the injected beam, with nonlinear detuning effect included, sufficiently approaches the 
resonance condition, $\nu_{x}-\nu_{y}-l=0$. The safe distance to the resonance condition is determined by the resonance strength $G$ defined in Eq. (3).

On the other hand, if the nonlinear detuning of the lattice is properly designed, it is possible to operate the storage ring with the linear working point right on the linear difference resonance, while the tune footprint of the injected beam avoids the difference resonance line. In this case, the horizontal oscillation will not be coupled to the vertical plane and no injection loss will occur due to the resonance. This principle can be applied to the design of diffraction limited storage rings (DLSR) to generate round beam with full coupling for the stored beam and in the same time use off-axis injection.

The experiments and simulation reveal an important point in the nature of linearly coupled beam dynamics in a nonlinear lattice. It is the nonlinear betatron tunes of the oscillating beam that determine its coupled motion, not the linear lattice tunes. Therefore, it is critical to include the nonlinear detuning in the analysis of the linearly coupled motion of realistic lattices.

\section{ACKNOWLEDGMENTS}

We thank James Safranek for helpful discussions. This work was supported by the U.S. Department of Energy, Office of Science, Office of Basic Energy Sciences, under Contract No. DE-AC02-76SF00515.

[1] L. Emery and M. Borland, in Proceedings of the 2003 Particle Accelerator Conference, Portland, OR (IEEE, New York, 2003), p. 256, TOPA014.

[2] A. Xiao, M. Borland, and C. Yao, in Proceedings of the 25th Particle Accelerator Conference, PAC-2013, Pasadena, CA, 2013 (IEEE, New York, 2013), p. 1076, WEPSM13.

[3] Y. Cai, K. Bane, R. Hettel, Y. Nosochkov, M.-H. Wang, and M. Borland, Ultimate storage ring based on fourth-order geometric achromats, Phys. Rev. Accel. Beams 15, 054002 (2012).

[4] R. Wanzenberg et al., Proceedings of IPAC 2017, Copenhagen, Denmark (2017), http://accelconf.web.cern .ch/AccelConf/ipac2017/papers/wepik065.pdf.

[5] R. Talman, A Proposed Möbius Accelerator, Phys. Rev. Lett. 74, 1590 (1995).

[6] P. Kuske and R. Grgen, in Proceedings of the 8th European Particle Accelerator Conference, Paris, 2002 (EPS-IGA and CERN, Geneva, 2002).

[7] M. Aiba et al., Proceedings of IPAC 2015, Richmond, VA, USA (2015), http://accelconf.web.cern.ch/AccelConf/ IPAC2015/papers/tupje045.pdf.

[8] I. Agapov, R. Brinkmann, J. Keil, and R. Wanzenberg, Noninterleaved round beam lattice for light sources, Phys. Rev. Accel. Beams 21, 051601 (2018).

[9] X. Huang, An ultimate storage ring lattice with vertical emittance generated by damping wigglers, Nucl. Instrum. Methods Phys. Res., Sect. A 777, 118 (2015).

[10] A. Xiao, L. Emery, V. Sajaev, and B. Yang, Proceedings of IPAC 2015 (2015), pp. 562-564, MOPMA013, http://accelconf.web.cern.ch/AccelConf/IPAC2015/papers/ mopma013.pdf.

[11] I. Agapov, Y.-C. Chae, J. Keil, G. Kube, A. I. Novokshonov, G. K. Sahoo, and R. Wanzenberg, Proceedings of IPAC 2018, Vancouver, BC, Canada (2018), pp. 14171419, TUPMF068, http://accelconf.web.cern.ch/AccelConf/ ipac2018/papers/tupmf068.pdf.

[12] S. Y. Lee, Accelerator Physics (World Scientific, Singapore, 1999).

[13] J. Safranek, Experimental determination of storage ring optics using orbit response measurements, Nucl. Instrum. Methods Phys. Res., Sect. A 388, 27 (1997).

[14] J. Safranek and X. Huang, in Proceedings of the 23rd Particle Accelerator Conference, Vancouver, Canada, 2009 (IEEE, Piscataway, 2009).

[15] A. Terebilo, in Proceedings of the 19th Particle Accelerator Conference, Chicago, IL, 2001 (IEEE, Piscataway, 2001).

[16] X. Huang, J. Safranek, and D. Dell'Orco, in Proceedings of the International Particle Accelerator Conference, Kyoto, Japan (ICR, Kyoto, 2010), p. 4627, THPE048. 